Review

\title{
Is the Concept of Photosynthetic Units Verified?
}

\author{
Yuzeir Zeinalov
}

Institute of Biophysics, Bulgarian Academy of Sciences, Academic G. Bonchev, str. bl. 21, Sofia 1113, Bulgaria. E-mail: zeinalov@bio21.bas.bg

Z. Naturforsch. 64c, 459-475 (2009); received February 2/May 4, 2009

In memory of Otto Warburg, Birgit Vennesland and Helmut Metzner

The cornerstones of the first fundamental concept of photosynthetic machinery, i.e. the concept of photosynthetic units, are reconsidered and a new logically and experimentally well sustained interpretation of the crucial observations of this hypothesis is presented. The results obtained lead to the conclusion that under low irradiances all chlorophyll molecules are in a state to perform charge separation (photochemical) reaction, while under increased irradiances the essential part of the reaction centres are blocked and only one reaction centre from 500-600 remains in functionally active state, i.e. in the range of the value obtained by the founders of the widely accepted concept of photosynthetic units.

Key words: Principles of Photosynthesis, Concept of Photosynthetic Units

\section{Fundamental Results}

In the beginning of the 4th decade of the last century, during the investigations of the photosynthetic light reactions, several important results were obtained, whose explanation led to significant complications and, thus, to the postulation of the first fundamental hypothesis, i.e. the concept of the photosynthetic unit (PSU) (Emerson and Arnold, 1932a, b; Gaffron and Wohl, 1936). According to this concept, in all photosynthetic systems (photosynthetic bacteria, green unicellular algae, higher plants etc.) the light-absorbing pigment molecules are divided into two groups. Only one highly specialized pigment molecule (named reaction centre) present among dozens of bacteria and among hundreds of green photosynthetic systems could carry out the photochemical (charge separation) reaction. The essential part of the pigment molecules absorbs light quanta only and transfers the light energy to the reaction centres.

In 1932, Emerson and Arnold applied for the first time flash-induced experiments in photosynthesis, using the manometric equipment introduced by Warburg (1920) and saturating flashing light, obtained by the discharge of an 1- or 0.5 $\mu \mathrm{F}$ condenser, charged to about $3000 \mathrm{~V}$, through a neon tube.

The fundamental results from the flash experiments of Emerson and Arnold were as follows:
1. The time required for one unit in the photosynthetic machinery to complete the cycle of photochemical and Blackman (dark) reactions was about $0.02 \mathrm{~s}$ at $25^{\circ} \mathrm{C}$.

2. The average flash yields were maximal when the spacing (dark period) between the flashes was about $0.02 \mathrm{~s}(20 \mathrm{~ms})$.

3 . The dependence of the quantity of the oxygen molecules (in mol) evolved after one saturated flash on the quantity of the chlorophyll (Chl) molecules in the Chlorella cell suspension was linear with a slope of about $1 \mathrm{O}_{2}: 2480$ chlorophyll molecules, thus suggesting that after every flash one oxygen molecule was produced by 2480 chlorophyll molecules. Consequently, in Chlorella pyrenoidosa suspensions with different chlorophyll concentrations approx. $4 \cdot 10^{-4} \mathrm{M}$ oxygen was evolved from $1 \mathrm{~mol}$ chlorophyll after every flash.

4. A good coincidence has been established between the experimentally obtained maximal rate of oxygen evolution, $P_{\max }$, under continuous saturated irradiation and the theoretically calculated maximal rate, $P=N / \tau$, where $N$ is the estimated number of the oxygen-producing units and $\tau$ is the turnover time from flash light experiments. This correlation has shown that under continuous saturated irradiation the number of effectively operating oxygen-evolving centres was approx. 1:600 of the number of the chlorophyll molecules in the investigated suspension, as estimated in the flash experiments. 
In 1932, besides these important observations, obtained by Emerson and Arnold, some additional facts had been also recognized:

5. The very high (maximum) quantum efficiency of photosynthesis under limited light intensity conditions, i.e. when the probability for light quanta absorption of a chlorophyll molecule was about 1 quantum per hour. This statement is a direct consequence of the investigations of the light (irradiance) dependence of photosynthesis. It has been shown in many experiments that the photosynthetic response to very low light intensities is linear. This type of light curves was accepted as a general rule.

6. The absence of an induction period in the process of oxygen evolution or carbon dioxide reduction under very low light intensity conditions: this means that at low irradiances photosynthesis starts before the absorption of four quanta, needed for the evolution of one $\mathrm{O}_{2}$ molecule, by separated chlorophyll molecules.

Using the equipment described in Zeinalov (2002) with a polarographic oxygen rate electrode and a group of four saturating short flashes, the dependence of the oxygen yields produced by separated flash groups on the spacing between the flashes in groups is presented in Fig. 1.

The data clearly show that the amplitudes and the volumes (quantity of oxygen) of the yields increased with increasing the spacing between

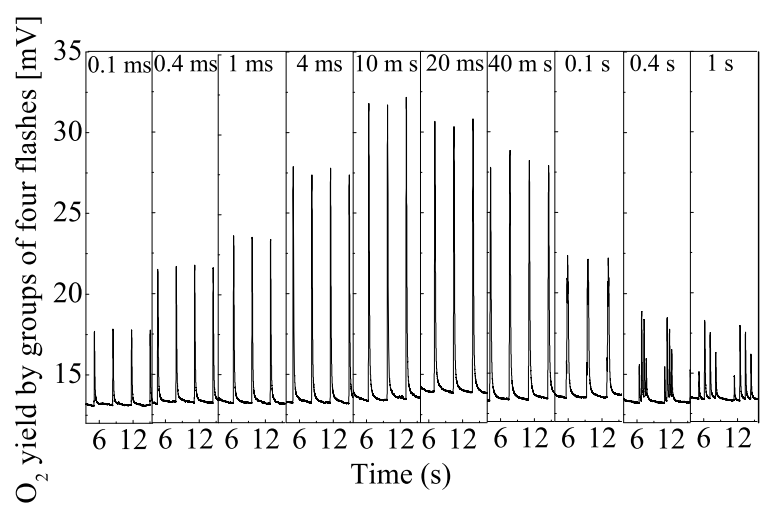

Fig. 1. The steady state oxygen yields of groups of four saturating flashes depending on the spacing (from $0.1 \mathrm{~ms}$ up to $1000 \mathrm{~ms}$, indicated on the graphs) between the flashes in the groups in a Scenedesmus obliquus suspension with 0.05 absorbance ( $0.1 \mathrm{ml}$ sample volume). The groups of 4 saturating flashes $\left(4 \mathrm{~J}, t_{1 / 2}=8 \mu \mathrm{s}\right)$ are spaced at $3 \mathrm{~s}$, and after reaching the steady state conditions the produced oxygen yields are presented. the flashes up to $10-20 \mathrm{~ms}$ and after that, the yields decreased. The results presented confirm the turnover time of the oxygen-evolving centres $\left(2 \cdot 10^{-2} \mathrm{~s}\right)$, estimated by Emerson and Arnold (1932a, b).

The decrease of the flash group yields with decreasing the spacing between the flashes in the groups below $10 \mathrm{~ms}$ could be explained with the time needed for accomplishment of the dark (Blackman) reaction and during this time, some of the centres were in the so-called "closed" state. The decrease of the yields with increasing the spacing between the flashes after $10-20 \mathrm{~ms}$ needs explanation that is more complicated. However, the most important reason, for the observed decrease, could be the deactivation of the higher oxidized $\mathrm{S}_{2}$ and $\mathrm{S}_{3}$ states, according to the model of Kok et al. (1970), with increasing the spacing between the flashes. This statement was supported by the yield of the first flash at the flash group yields with 400 and $1000 \mathrm{~ms}$ of spacing (see Fig. 1). Another possible reason could be the respiration and the anaerobic condition of the suspension and the cell volumes.

Five oxygen induction curves recorded at different irradiances after 3 min of dark adaptation of a Chlorella pyrenoidosa cell suspension are presented in Fig. 2a. Curve A was recorded at maximum irradiance, $I_{0}=135 \mathrm{~W} \mathrm{~m}^{-2}$, corresponding to the oxygen evolution rate close to satura-

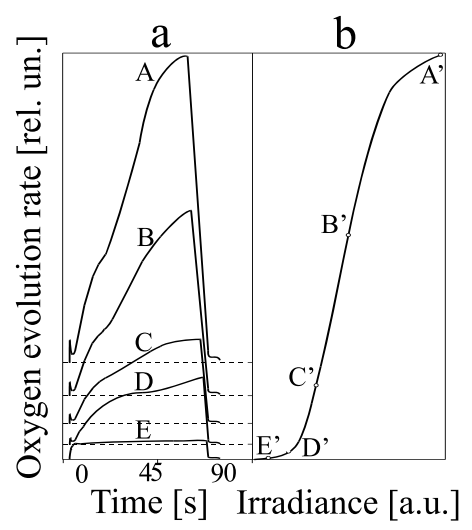

Fig. 2. (a) The oxygen induction curves recorded at different irradiances after 3 min of dark adaptation and (b) the respective "operating points" on the light curve in a Chlorella pyrenoidosa suspension with 0.05 absorbance. The induction curves $\mathrm{A}, \mathrm{B}$ and $\mathrm{C}$ are recorded at $8 \cdot 10^{-8} \mathrm{~A} / \mathrm{mm}$ and the curves $\mathrm{D}$ and $\mathrm{E}$ at $1.2 \cdot 10^{-9} \mathrm{~A} /$ $\mathrm{mm}$ sensitivity of the polarograph. 
tion (see Fig. 2b, point $A^{\prime}$ ). The other curves were recorded at $0.76 I_{0}(\mathrm{~B}), 0.46 I_{0}(\mathrm{C}), 0.19 I_{0}(\mathrm{D})$, and $0.056 I_{0}(\mathrm{E})$, respectively.

The induction curves indicate that the duration of the induction period decreases simultaneously with decreasing irradiance and under the lowest one $-0.056 I_{0}(\mathrm{E})$-, the rate of oxygen evolution reaches its steady state immediately after the light was switched on. Thus, data in this figure confirm the statement that under very low irradiance conditions photosynthesis starts without any appreciable induction time.

The following two very important observations were obtained significantly later and are considered as additional confirmation and verification of the concept of the PSU:

7. The investigations of Alen and Franck (1955), Whittingham and Brown (1958), and of Joliot (1961) had shown that after approx. 5 min of dark adaptation of unicellular algae (e.g. Chlorella, Scenedesmus), or isolated chloroplast suspensions, the oxygen yield of the first saturating short (10 $\mu$ s) flash was zero.

8 . The oxygen flash yields were oscillating with a period of four flashes observed after 5-6 min of dark incubation in alga or chloroplast suspensions (Joliot et al., 1969).

As we will see in the next section, a logical interpretation of these observations is extremely complicated and contradictory!

\section{Problems and Hypotheses}

In many textbooks and manuals the integral equation of photosynthesis is presented as

$$
\mathrm{H}_{2} \mathrm{O}+\mathrm{CO}_{2}+\text { light energy } \rightarrow\left(\mathrm{CH}_{2} \mathrm{O}\right)+\mathrm{O}_{2} \text {. }
$$

The standard free energy for the synthesis of one mol of $\mathrm{CO}_{2}$ to the level of carbohydrate $\left(\mathrm{CH}_{2} \mathrm{O}\right)$ is $112.5 \mathrm{kcal} / \mathrm{mol}$ supplied by the light quanta. Considering the general equation of photosynthesis, it is apparent that for the evolution of one oxygen molecule or for the reduction of one carbon dioxide molecule to the level of carbohydrate four electrons should be transferred on account of the absorbed light quanta energy and, consequently, at least four photons are needed. The following principal questions for understanding and explanation of the observed experimental results arise: (i) Are energy or photoproducts (oxygen precursors) of the absorbed four photons summarized?

(ii) Whether oxygen-evolving centres act independently of each other, or can exchange energy, or whether the oxygen precursors (positive charges?) could migrate and cooperate in the surrounding medium?

The estimations have shown that the quantum efficiency in thick (optically dense) suspensions is maximal under low irradiance in the range of $100-1000 \mathrm{~lx}$ or about $10^{14}-10^{15}$ photons $\mathrm{cm}^{-2} \mathrm{~s}^{-1}$. It is well known that the average effective cross section for light quanta absorption of a chlorophyll molecule in a solution is ca. $0.2 \cdot 10^{-16} \mathrm{~cm}^{2}$. This means that under low irradiances, i.e. $10^{13}-10^{15}$ photons $\mathrm{cm}^{-2} \mathrm{~s}^{-1}$, the time needed for absorption of four quanta by separated chlorophyll molecules should be ca. $1 \mathrm{~h}$. Obviously, under such conditions, if the oxygen-evolving centres act independently of each other, the photosynthetic oxygen evolution should start after a prolonged induction time, which is in contradiction with the observation 6 and the results presented in Fig. 2, showing that in reality photosynthesis starts immediately without any induction period. The existence of the optimal spacing between the flashes $(0.02 \mathrm{~s})$, leading to the maximal oxygen flash yield estimated in the experiments of Emerson and Arnold (see also the results presented in Fig. 1), shows that if the spacing between flashes is increased, the oxygen flash yields decrease which is in contradiction with the accepted linear light dependence of photosynthesis. It is easy to understand that the increase of the spacing between the flashes, or the decrease of the frequency of the flashes is identical with the decrease of the continuous irradiance intensity. It is evident that under low irradiance of the continuous irradiation the photosynthetic oxygen-evolving units receive quanta after prolonged intervals, which is analogous to the increase of the spacing between the flashes. Thus, the decrease of the oxygen flash yields with the increase of the spacing between the flashes should lead to a decrease in the efficiency of the continuous irradiation with low intensities and consequently to the non-linearity of the light curves under such conditions. However, the nonlinearity of the photosynthetic light curves, which arises from the analysis of the flash-induced experiments, are in contradiction with the accepted, 
at that time linear, dependence of photosynthesis under very low irradiances.

The observation 2 , as well as the additional observations 7 and 8 lead to the conclusion that the oxygen-evolving centres operate independently of each other (non-cooperative mechanism). This means that every oxygen-evolving centre, before producing one oxygen molecule, should accept four light quanta (photons). The observed results could be explained if we suppose that the oxygen-evolving reaction centres are in a state to conserve some of the $\mathrm{O}_{2}$ precursors (e.g. positive charges) for several minutes or even hours and, upon following illumination after the absorption of the first photons, they could start evolving oxygen immediately. This assumption, however, is in contradiction with the observations 2,7 and 8 showing that the oxygen precursors are deactivated and the effect of the light quanta disappears in the dark. Hence, we should conclude that the oxygen precursors are unstable in the dark and deactivate almost entirely for about $100 \mathrm{~s}$.

If the oxygen precursors are unstable in the dark, the observed results, i.e. the absence of prolonged induction time and the high quantum efficiency of photosynthesis under low light intensities, could be explained by the assumption that even under limited light conditions the oxygen-evolving centres receive photons for time intervals of about seconds or even shorter. This assumption could be explained by an additional speculation that hundreds of chlorophyll molecules are functionally or even structurally assembled around a given specialized chlorophyll molecule (named reaction centre) which carries out the photochemical reaction, and that this centre is supplied with the photons absorbed by the assembled light-harvesting (antenna) molecules. In this way the effective cross section of the light quanta absorption of the reaction centre molecule is increased by hundreds of times, and even under very low light intensity conditions the reaction centres receive the needed four quanta for intervals hundreds of times shorter than the intervals of the separated chlorophyll molecules. This assumption explains both the absence of the prolonged induction period and the high quantum efficiency under low light intensities. In agreement with this assumption is the observation 3, i.e. the ratio $\mathrm{O}_{2} / \mathrm{Chl}$ per flash, showing that after every saturating flash only one oxygen molecule is produced from approx. 2500 chlorophyll molecules. This attractive hypothesis had been postulated by Gaffron and Wohl (1936) and was immediately accepted by the investigators. Since then it has been supported by a great number of investigations, and especially by the findings 7 and 8. Initially it was assumed that approx. 600 chlorophyll molecules are organized in a special structure and, after accepting one light quantum, this structure transfers the quantum energy to the specialized reaction centre molecule. (The number of the molecules - 600 - is obtained after dividing the number 2400 by 4 , as for evolution of one oxygen molecule four electrons should be transferred, i.e. the energy of four light quanta should be used.) At that time the photosynthetic units had been presented as a funnel filled with so-called antenna chlorophyll molecules and with a reaction centre molecule situated in the neck of the funnel. The picture was changed with accepting the concept of two photosystems.

It was supposed that two different photosystems should have different photosynthetic units with different antenna chlorophyll molecules. So, it was accepted that the number of chlorophyll molecules in the two types of PSUs should decrease to 300 . Nevertheless, a new problem connected with the distribution of the absorbed light quanta at different wavelengths between the two types of PSUs emerged. So it was assumed that there exists a special structure, called "light-harvesting complex" (LHC), that is able to divide in equal shares between the units the energy of the absorbed light quanta. In turn, this complication was also insufficient and it was supposed that photosystem II (PSII) reaction centres have a separate light-harvesting complex, i.e. "lightharvesting complex II" (LHCII). A series of theoretical considerations and complicated models of the structure of PSUs has been considered over the years. However, a significant number of investigations have shown a lot of discrepancies concerning the size and the structure of the postulated PSUs (Tumerman and Sorokin, 1967; Schmidt and Gaffron, 1971; Lavorel and Joliot, 1972). According to Mauzerall and Greenbaum (1989) the answer to the simple question "how large is the PSU?" is rather complicated and variable. This leads to the more ticklish question: Are the above considered basic arguments sufficient for the postulation of the concept of the PSU? Are there other possibilities for explanation of the obtained observations? A careful analysis of these arguments has shown that our difficulties 
with the logical explanation of the results considered above arise from the observations 2, 7 and 8 , i.e. from the absence of the oxygen burst or oxygen yield at the first flash given after several minutes of dark incubation, and from the oscillations of the flash yields with a periodicity of four flashes. These observations support the hypothesis that the oxygen-evolving centres are functioning independently and every oxygen-evolving centre evolves one oxygen molecule after the absorption of four quanta consecutively. So, we have rejected any cooperativeness in the action of the oxygenevolving centres. Obviously, the existence of the non-cooperative mechanism of oxygen evolution in photosynthesis has been completely confirmed with the observations 7 and 8 and in numerous flash experiments, as well. More specifically, the model of Kok et al. (1970) or the $\mathrm{S}_{i}$ states model, in which only the non-cooperative mechanism is supposed, could explain both the absence of the oxygen flash yield at the first saturating flash after prolonged dark incubation and the oscillations in the oxygen flash yields with a period of four flashes.

Regardless of this, a number of kinetic models have been proposed for explanation of various complicating phenomena of the oxygen flash yield oscillations (Delrieu, 1981; Lavorel and Lemasson, 1976; Lavorel and Maison-Peteri, 1983). According to Lavorel $(1976,1980)$ a special kind of cooperative action exists in the functioning of the $\mathrm{S}_{i}$-states. In addition, there are some experimental results, which cannot be explained by Kok's model (e.g. the linearity of the light curves under very low irradiance conditions). As it was shown (Zeinalov, 1977) the operation of Kok's model leads to non-linear, "S"-shaped light curves. On the other hand, it is evident that the existence of the non-cooperative mechanism of oxygen evolution does not exclude the participation and existence of the cooperative one. Furthermore, the absence of the oxygen flash yield after the first flash cannot be considered as a proof for the absence of cooperativeness in the oxygen evolution because of the following reasons:

1. The first flash was applied after prolonged (5-10 min) dark incubation of the algae or chloroplast suspensions, thus leading to anaerobic conditions in the cell and chloroplast volumes.

2. Since the functioning of the cooperative mechanism should be realized by the diffusion of oxygen precursors, produced in different oxygen- evolving centres, the rate constant of the reactions leading to oxygen evolution through the cooperative mechanism should be significantly lower than the rate constant of the non-cooperative one.

Consequently, it could be concluded that the registration of oxygen burst or oxygen production by the first flash will be difficult and even impossible. In addition, if we consider the observation 5, i.e. the linearity of the light curves under low light intensity conditions, and reasons 1 and 2 , i.e. the dependence of yields on the dark intervals between the flashes, as well as the absence of the oxygen yield at the first flash, it is reasonable to conclude that these observations are mutually contradictory. If the observations 1 and 2 reflect strictly the photosynthetic $\mathrm{O}_{2}$ production upon flash irradiation, then even with structures like the postulated PSUs the light curves of photosynthesis (oxygen evolution) should have a non-linear part under very low light intensity conditions. This means that, independently of the existence of the PSUs, the light curves of photosynthesis should be S-shaped, if one supposes that the oxygen production is realized only through the non-cooperative mechanism and that defined deactivation reactions exist. Thus, two possibilities could be considered:

(a) the cooperative mechanism is also functioning simultaneously with the non-cooperative one;

(b) the light curves of photosynthesis exhibit a non-linear part at very low light intensity conditions.

The first assumption gives a logical explanation of the basic arguments which have led to the postulation of the concept of the PSU, i.e. the observations 5 and 6 , while the observations 7 and 8 can be explained with the functioning of the noncooperative mechanism. The observations 3 and 4 will be reconsidered in the next section. If we accept the second possibility, then we can explain the "red drop" and "enhancement" effects of Emerson, which are considered the cornerstones in the concept of the two photosystems, without using this concept. Our investigations during the last 30 years have shown that these two possibilities are accomplished. Therefore, despite the participation of the cooperative and non-cooperative mechanisms of photosynthetic $\mathrm{O}_{2}$ evolution, the irradiance dependence of photosynthesis is a nonlinear function, i.e. the light curves are S-shaped. Probably under low irradiance conditions, a sig- 
nificant part of the photosynthetically evolved oxygen is consumed by the dark respiration and, under such conditions, the registered light curves are with low slopes and the quantum efficiency is also low.

The following observations could be considered in favour of the cooperative mechanism:

1. In unicellular alga suspensions a prolonged (5-20 min) oxygen evolution is registered after switching off the continuous irradiation.

2 . The decay kinetics in the oxygen flash yields is at least biphasic, i.e. two different processes leading to the oxygen production exist - non-cooperative and cooperative mechanisms.

3. We also cannot explain the absence of the induction period under low irradiances without participation of the cooperative mechanism.

4. In some photosynthetic systems (cyanobacteria) we cannot register any oxygen flash yields, although they can produce oxygen at a high rate under continuous irradiation.

5. Our previous studies (Lehoczki and Zeinalov, 1984; Maslenkova et al., 1989) did stress the fact that the non-cooperative oxygen-evolving mechanism is operating mainly in the grana regions while the cooperative one is localized predominantly in the stroma thylakoids.

The existence of the two different oxygenevolving mechanisms - cooperative and non-cooperative - situated in grana and stroma thylakoids puts the question about the realization of these mechanisms. Another interesting question is the localization of the two kinds of bound $\mathrm{CO}_{2}$ (or $\mathrm{HCO}_{3}$ ) (Stemler, 1980a, b) in chloroplast stroma and grana thylakoids.

\section{Variation in the Number of Effectively Func- tioning Oxygen-Evolving (Reaction) Centres and the First Mistake Made by Emerson and Arnold}

Let us suppose that a suspension of unicellular algae (Chlorella, Scenedesmus etc.) contains $N_{0}$ reaction centres (RCs). Let us suppose $\tau$ be the average turnover time of these centres as determined by the rate of the limiting dark reaction. Then the maximum rate $\left(P_{\max }\right)$ of photosynthesis (i.e. the maximum number of $\mathrm{O}_{2}$ molecules evolved per second) could be given by the equation

$$
P_{\max }=k N_{0} / \tau,
$$

where $k$ is a constant factor, which, in case of $\mathrm{O}_{2}$ evolution or $\mathrm{CO}_{2}$ reduction, is equal to 0.25 . If photons arrive at the reaction centres within a time $T<\tau$, they cannot be effectively used for photochemical processes. If, on the other hand, $T>\tau$, the centres will remain idle during the time $T-\tau$. In the latter case the rate of photosynthesis will be given by the expression

$$
P=k N_{0} / T \text {. }
$$

In this case a part, i.e.

$$
N=N_{0}(T-\tau) / T,
$$

of the centres (at any given time) will be in an inoperative (open) state. Under low irradiance conditions, i.e. if $T>>\tau$, the number of these inoperative centres will tend to $N_{0}$, i.e. to the total number of the centres in the sample. Under saturating irradiance conditions, i.e. $T \leq \tau$ the number of inoperative centres will, on the contrary, tend to zero. By submission of $\tau$ and $T$ in (3), using (1) and (2), the following relation between $N, N_{0}, P_{\max }$ and $P$ will be obtained:

$$
N=N_{0}-N_{0} \cdot P / P_{\text {max }}
$$

Obviously, the $N$ vs. $P$ plot is a straight line (Fig. 3, curve c), crossing the ordinate at $N=N_{0}$ and the abscissa at $P=P_{\max }$.

The experimental determination of the ratio between total and open (inoperative) centres is relatively easy. According to the model of Kok et al. (1970) the oxygen-evolving centres exist in five different oxidized states: $\mathrm{S}_{0}, \mathrm{~S}_{1}{ }^{+}, \mathrm{S}_{2}{ }^{2+}, \mathrm{S}_{3}{ }^{3+}$, and $\mathrm{S}_{4}{ }^{4+}$. Every centre which absorbs one photon passes to the next higher oxidized state. After reaching the state $\mathrm{S}_{4}{ }^{4+}$, one oxygen molecule is produced, and the centre returns to the initial $S_{0}$ state. It is easy to understand that, independently of the oxidation state, every centre after absorption of four photons separated by dark intervals equal to or longer than the turnover time $(20 \mathrm{~ms})$ will evolve one oxygen molecule and attain its former state. Consequently, the amplitudes of the oxygen bursts produced by four saturating flashes will reflect the number of the centres in an inoperative (open) state. This means that, if the flash groups are supplied in the darkness (all centres are open), the amplitudes of the bursts will reflect the total number of the centres.

The results obtained with Chlorella pyrenoidosa cells, using excitation with groups of four saturating flashes $\left(t_{1 / 2}=8 \mu \mathrm{s}\right)$ spaced by $20 \mathrm{~ms}$ between each other and $7 \mathrm{~s}$ of dark intervals between the 
groups on the background of gradually increasing continuous irradiation with achromatic (white) light, are depicted in Fig. 4. The data show that, in contrast to our expectations, the amplitudes of the oxygen bursts produced by the group of flashes in the darkness (0) were remarkably small and, after applying continuous irradiation (curves 1,2, 3 , and 4), a significant increase could be observed. With increasing the intensity of the background continuous irradiation (curves 5, 6, and 7), the amplitudes of the oxygen bursts decreased and, after reaching the saturated background irradiation (curve 7), they were almost invisible.

The relationship between the amplitudes and the steady state oxygen evolution is presented in Fig. 3. Curve a was obtained by increasing the background irradiation from zero to saturation level. Curve b was drawn for the reverse direction, i.e. with gradually decreasing background irradiation. Obviously, the difference between the two curves reflected a "hysteresis" effect most probably due to the induction phenomena in the photosynthetic process. It should be pointed out that the shape of the curves $a$ and $b$ presented in Fig. 3 was dependent on the experiment duration as well as the preceding history of the investigat-

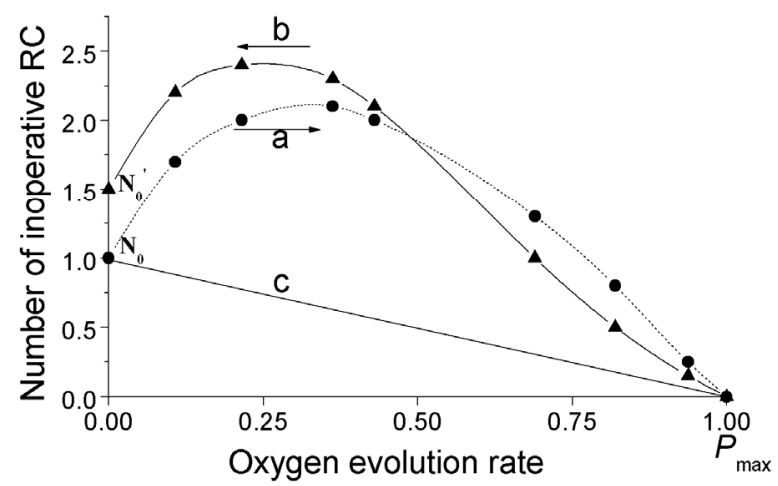

Fig. 3. The number of inoperative (open) centres in Chlorella pyrenoidosa, depending on the oxygen evolution rate level. (a) Experimentally obtained results by increasing the light intensity of background irradiation from 0 to the saturation level $\left[\mathrm{O}_{2}\right.$ rate from 0 to the maximal $\left(P_{\max }\right)$ rate], $(\mathrm{b})$ in opposite direction, and (c) straight line, predicted by the theory of the photosynthetic unit concept after (4). ed alga suspensions. Nevertheless, an unexplainable difference between the theoretically predicted (on the basis of the concept of the PSU) straight line $\mathrm{c}$ and the curves $\mathrm{a}$ and $\mathrm{b}$ still remains. The amplitudes of the oxygen burst increased under background irradiation; they reached their maximum at the level of steady state oxygen evolution rate, representing approx. $1 / 3-1 / 2$ of the maximum value of the saturating level.

Whenever flash groups are given under low irradiance, the lower value of the amplitudes reflects the existence of induction phenomena. It is obvious that we cannot estimate the exact number of reaction centres from the amplitudes of oxygen yield under dark conditions, i.e. without background irradiation.

The results presented in Fig. 5 were obtained using a suspension of unicellular algae (Chlorella pyrenoidosa) irradiated with groups of four short $\left(t_{1 / 2}=10 \mu \mathrm{s}\right)$ saturated (4 J) flashes, spaced by intervals of $20 \mathrm{~ms}$ and with $5 \mathrm{~s}$ between the groups. In the first $20 \mathrm{~s}$ the amplitudes of the oxygen yields from the groups of flashes in the darkness are shown. Then a saturated, continuous white (achromatic) light was switched on and the so-called induction phenomenon started. After an initial

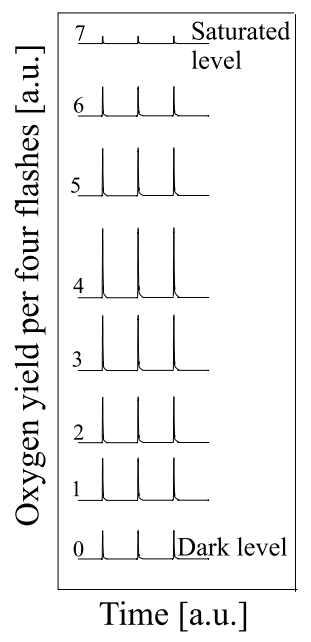

Fig. 4. The amplitudes of the oxygen yields in Chlorella pyrenoidosa, produced by four saturating flashes $\left(4 \mathrm{~J}, t_{1 / 2}=8 \mu \mathrm{s}\right)$ with $20 \mathrm{~ms}$ of dark periods between the flashes and $7 \mathrm{~s}$ between the groups depending on the steady state oxygen evolution rate. The intensities of background light are: 0,$0 ; 1,17.0 ; 2,25.0 ; 3,34.0 ; 4,43.0$; $5,52.0 ; 6,82.0 ; 7,135.0 \mathrm{~W} \mathrm{~m}^{-2}$. 


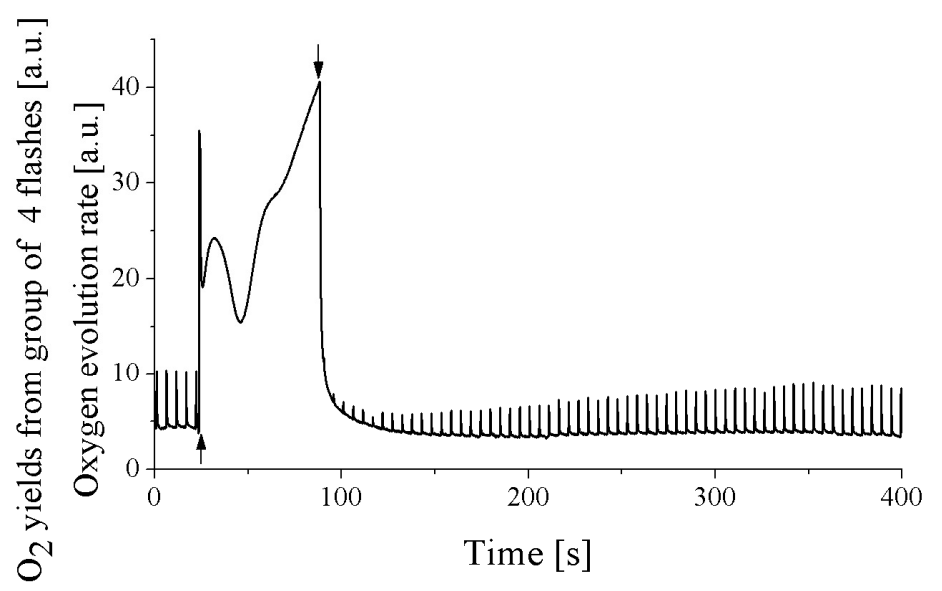

Fig. 5. The variations in the oxygen bursts before, during and after the induction time of photosynthesis in Chlorella pyrenoidosa, registered with an oxygen rate electrode as described in Zeinalov (2002). The suspension of cells is kept in the darkness for $5 \mathrm{~min}$ and the groups of four saturating flashes $(20 \mathrm{~ms}$ spacing between the flashes and $5 \mathrm{~s}$ between the groups) are switched on at the time " 0 ". The saturated white light $\left(135 \mathrm{~W} \mathrm{~m}^{-2}\right)$ is switched on at the time indicated by "^" and is switched off at the time indicated by " $\downarrow$ ".

oxygen burst and some oscillations, an approx. maximal (saturated) oxygen evolution rate was reached, and the continuous light was switched off. The results presented in Fig. 5 demonstrated a well expressed variation in the $\mathrm{O}_{2}$ yields from flash groups. As the spacing between the flashes in the flash groups was $20 \mathrm{~ms}$, i.e. equal to the Emerson and Arnold period, the amplitudes of the yields should reflect the number of open reaction centres (oxygen-evolving centres). The initial amplitudes (in the dark, before switching on the continuous light) of the oxygen bursts could be considered proportional to the total number of the oxygen-evolving centres in the suspension, because all the centres are in open state. During the time of irradiation with continuous saturated light, the amplitudes of the oxygen burst were zero, indicating that all oxygen-evolving centres were closed, i.e. in operative state. It is easy to understand that, after switching off the light after the first $10-20 \mathrm{~ms}$, all reaction centres should be in open state, i.e. the amplitudes of the oxygen yields induced by the four flashes should be equal to the amplitudes before switching on the continuous irradiation! In contrast to our expectation, the amplitudes of the flash groups immediately after switching off the continuous irradiation are almost invisible and they increased in the darkness at prolonged time (more than $5 \mathrm{~min}$ ), thus indicating that during the induction time (during the irradiation with continuous light) the essential part of the oxygen-evolving centres was inactivated or blocked.

The next two figures give some additional ideas about the kinetics of blocking the oxygenevolving centres during the induction time of photosynthesis. The oxygen bursts presented in Fig. 6 were obtained with a Chlorella pyrenoido$s a$ suspension excited with groups of four flashes, spaced by $20 \mathrm{~ms}$ (Emerson and Arnold period) and $2 \mathrm{~s}$ between the groups. At the time $t_{1}$ continuous light with $650 \mathrm{~nm}$ wavelength and $400 \mu \mathrm{mol}$ photons $\mathrm{m}^{-2} \mathrm{~s}^{-1}$ was switched on only for $30 \mathrm{~ms}$. For this short time, every oxygen-evolving centre could perform no more than one or two turnovers (the turnover time of the centre is around $20 \mathrm{~ms}$ ), so that oxygen could not be produced by a noncooperative (Kok's) mechanism (at least 3 or 4 turnovers are needed for producing one oxygen molecule). Nevertheless, the appearance of the initial oxygen burst showed that the induction of oxygen production is started, most probably according to the cooperative mechanism. A comparison of the amplitude of the first oxygen burst, after switching off the continuous irradiation, with the amplitudes of the flash group yields before switching on the continuous irradiation (i.e. in the darkness) shows that they are equal. This means 


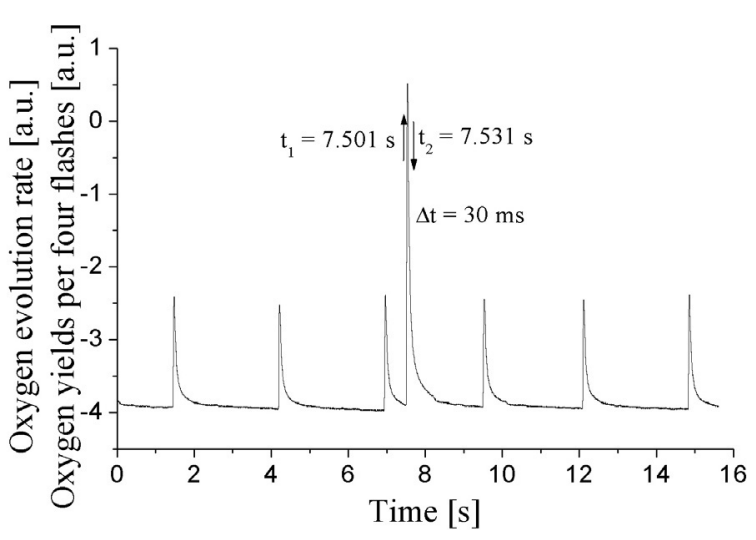

Fig. 6. Changes of the amplitudes of oxygen bursts produced by groups of four saturating $\left(4 \mathrm{~J}, t_{1 / 2}=8 \mu \mathrm{s}\right)$ flashes with $20 \mathrm{~ms}$ of dark periods between the flashes and $2 \mathrm{~s}$ between the groups after the induction time of photosynthesis. At the time $t_{1}$ the suspension of Chlo-

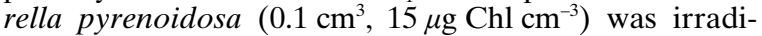
ated with a $650-\mathrm{nm}$ monochromatic light beam with $400 \mu \mathrm{mol}$ photons $\mathrm{m}^{-2} \mathrm{~s}^{-1}$ for $30 \mathrm{~ms}$. The upward and downward arrows show the times of switching on and off the continuous irradiations.

that the concentration of the produced oxygen in the cell volumes is below the inactivation starting level of the oxygen-evolving centres.

In Fig. 7 the time of irradiation with continuous light was increased up to $288 \mathrm{~ms}$. Even after such a relatively short time of irradiation, for which every oxygen-evolving centre could perform only 14 turnovers $(14 \cdot 20 \mathrm{~ms}=280 \mathrm{~ms})$, the amplitude of the oxygen flash group yields after switching off the red light decreased drastically. Consequently, during this short time of irradiation $(288 \mathrm{~ms})$ with not so strong continuous light the essential part of the oxygen-evolving centres was submitted to the process of blocking.

Let us consider the results presented in Fig. 8, showing that the effects of the flash groups on the background saturating "white light" are negligible. At the time $t=0$ the "white light" was switched off and the rate of oxygen evolution decreased sharply to the level indicated by D. After that, the process of oxygen evolution in the darkness, connected with deactivation of the $S_{i}$ states (Zeinalov and Litvin, 1979) or with the unblocking of the inactivated (blocked) states, began. Immediately after switching off the continuous saturated radiation, the effect of the flash groups was very small and the amplitudes of oxygen yields increased

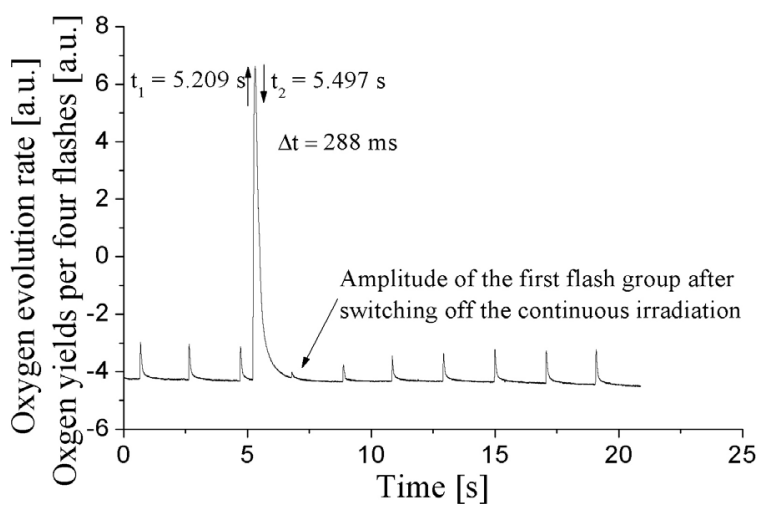

Fig. 7. The same experiments as shown in Fig. 6, except that the time of irradiation with continuous light is increased $(288 \mathrm{~ms})$.

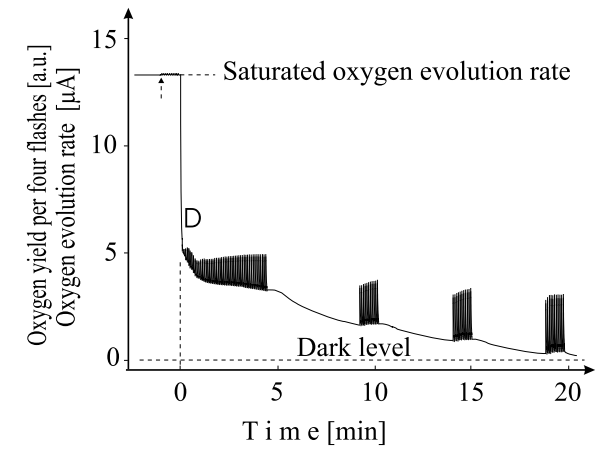

Fig. 8. The oxygen bursts produced by groups of four saturating $\left(4 \mathrm{~J}, t_{1 / 2}=8 \mu \mathrm{s}\right)$ flashes with $20 \mathrm{~ms}$ of dark periods between the flashes and $7 \mathrm{~s}$ between the groups. The suspension of Chlorella pyrenoidosa $\left(0.1 \mathrm{~cm}^{3}, 15 \mu \mathrm{g}\right.$

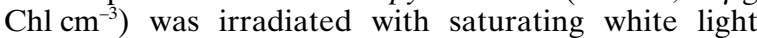
$\left(135 \mathrm{~W} \mathrm{~m}^{-2}\right)$ and at the time indicated as " 0 " the saturating light was switched off. The groups of flashes were switched on at the time indicated by “个”.

slowly in the dark up to $20 \mathrm{~min}$. Consequently, the increase of the amplitude of oxygen group yields in the darkness [after switching off the background radiation, when all the centres (after 10-20 ms) should be in open state] showed that the number of effectively working oxygen-evolving centres increased. This number was significantly lower (tending to " 0 ") immediately after switching off the saturated background radiation. Thus, we might suppose that this number had the same low value during the preceding time of irradiation with saturating "white light". This means that un- 
der saturating irradiance conditions, the essential part of the RC was in an inactivated (blocked!) state. More than 15 years after publication of our results (Zeinalov, 1986) the Hungarian scientists Scilard and coworkers (2002) showed that light flashes could inactivate the oxygen-evolving capacity of PSII. The results presented in Figs. 5-8 as well as in our paper (Zeinalov, 1986) show that the oxygen-evolving reaction centres are blocked not only under flash, but also under continuous irradiation.

According to Boitchenko and Efimtcev (1979) a significant part of oxygen-evolving (PSII) centres is inactivated (blocked) under increased oxygen concentration. Shinkarev (2005) accepted the state of inactivation (blocking) for all $S_{i}$ states in his model for photosynthetic oxygen-evolving reactions.

It is easy to show that the relation between the number of operating reaction centres $\left(N_{\mathrm{c}}\right)$, the amperometric current $(I)$ on a polarograph equipped with an oxygen rate electrode, the turnover time of the reaction centres $(\tau)$, and the electric charge of the electron $e=1.602189 \cdot 10^{-19} \mathrm{C}$ is as follows:

$$
N_{\mathrm{c}}=I \tau / e .
$$

If we accept for the turnover time of the centres the value by Emerson and Arnold (1932a, b) $-2 \cdot 10^{-2} \mathrm{~s}-$ and for the amperometric current of the saturated $\mathrm{O}_{2}$ evolution rate the value from Fig. $8-1.32 \cdot 10^{-5} \mathrm{~A}$ - we can calculate the number of the oxygen-evolving centres $N_{\mathrm{c}}$ in the investigated sample:

$$
\begin{aligned}
N_{\mathrm{c}} & =I \tau / e \\
& =1.32 \cdot 10^{-5} \mathrm{~A} \cdot 2 \cdot 10^{-2} \mathrm{~s} / 1.6 \cdot 10^{-19} \mathrm{C} \\
& =1.65 \cdot 10^{12} \mathrm{RC} .
\end{aligned}
$$

The comparison between the number of chlorophyll molecules $\left(N_{\mathrm{Chl}}\right)$ in the investigated sample $\left(0.1 \mathrm{~cm}^{3}\right.$ with $15 \mu \mathrm{g} \mathrm{Chl} \mathrm{cm}{ }^{-3}$, i.e. $9.4 \cdot 10^{14}$ chlorophyll molecules) and the number of oxygenevolving centres $\left(N_{\mathrm{c}}\right)$ leads to

$$
\begin{aligned}
N_{\mathrm{Chl}} / N_{\mathrm{c}} & =9.4 \cdot 10^{14} \mathrm{Chl} / 1.65 \cdot 10^{12} \mathrm{RC} \\
& =570 \mathrm{Chl} / \mathrm{RC} .
\end{aligned}
$$

If the number of chlorophyll molecules is calculated for one oxygen molecule evolved, the obtained value should be increased by 4 times, i.e. about 2280 for $1 \mathrm{O}_{2}$ molecule. Consequently, the value obtained in this way is in accordance with the value for the PSU proposed by Emerson and Arnold (1932a, b).
From the results presented in Fig. 8 it could be concluded that the number $N_{\mathrm{c}}$ estimated above reflects only the number of effectively working $\mathrm{RC}$ under saturating irradiance conditions, but not their total number. An approximate idea about the total number of the oxygen-evolving centres could be obtained if we compare the amplitudes of the oxygen yields per four flashes (Fig. 8) immediately after switching off the saturating "white light" with those obtained 20 min after switching off the light. Approx. a 30- to 40-times increase was registered after switching off the light. Having in mind the results presented in Figs. 3 and 4, indicating an increase of the amplitudes of oxygen flash yields up to 2-3 times under moderate irradiation with continuous light, we should increase these numbers up to 60-120. So, these numbers show that the number of unblocked, effectively operating oxygen-evolving centres under saturating irradiance conditions was 60-120 times lower than the maximum value of the centres shown in Fig. 3. However, this maximum did not reflect the total number of the oxygen-evolving centres. It is evident that the maximum value of the oxygenevolving centres shown in this figure represented only a balance between the two different processes:

1. the process of blocking (curve a) or unblocking (curve b) of the oxygen-evolving centres under higher light intensities (or at a higher oxygen concentration in the cell or chloroplast volumes), and

2. the process of oxygen consumption by dark respiration and the so-called induction phenomena, leading to a decrease of the oxygen burst amplitudes produced by the four saturating flashes.

If the second process was not taking part, the two curves ( $a$ and $b$ ) presented in Fig. 3 under low irradiances, tending to "0", probably would continue to the top of the graph, and the considered total number of oxygen-evolving centres should be increased at least two times, i.e. to 120-240. As we have shown the number of chlorophyll molecules in the operative RCs under saturating irradiance conditions is in the order of 500; this means that the total number of RC could be practically equal to the number of chlorophyll molecules. This indicates that the usual procedures used for estimation of the number of PSUs have to be revised. There are mainly two reasons for this statement: 
1. Under high light intensity or frequency of the saturating flashes the oxygen flash yields are low due to the inactivation (blocking) of the essential part of the RC.

2. Under low light intensity conditions, the oxygen flash yields are low as a consequence of the induction phenomena and the process of blocking (connected with oxygen consumption) of the essential part of the oxygen-evolving centres.

We have found (Zeinalov, 1979) that after switching on the irradiation during the induction time of photosynthesis, oxygen absorption reactions connected with the oxidation (blocking) of oxygen-evolving centres take place. The amount of oxygen absorbed (Fig. 9, dashed area A) during the induction time is in the order of the chlorophyll content and approx. the same amount of oxygen (Fig. 9, dashed area B) is evolved after switching off the light (in the darkness).

On the other hand, according to Emerson and Lewis (1941a, b) and McAlister (1939) the amount of $\mathrm{CO}_{2}$ burst during the induction period is also in the order of the amount of chlorophyll, which was explained by Franck and Herzfeld (1941) as a result of decomposition of the so-called $\mathrm{ACO}_{2}$ complex ( $\mathrm{A}$ is the primary acceptor of $\mathrm{CO}_{2}$ whose quantity according to these authors was equal to the amount of chlorophyll!) in the light. According to the "biochemical hypothesis" of Franck and Herzfeld, the absorption of a photon by any chlorophyll molecule leads to the formation of a

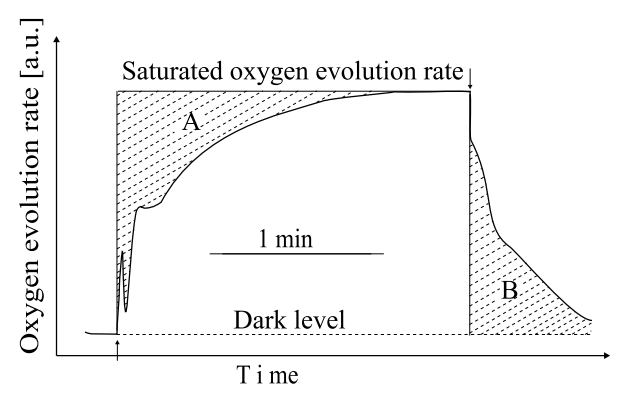

Fig. 9. The induction curve of photosynthesis of Chlorella pyrenoidosa, recorded after $5 \mathrm{~min}$ of dark incubation and after irradiation with $135 \mathrm{~W} \mathrm{~m}^{-2}$ "white light". " $\uparrow "-$ light on; “ $\downarrow "$ - light off. For details, see text. The number of $\mathrm{O}_{2}$ molecules absorbed during the induction time of photosynthesis, calculated from the dashed area "A" and evolved after switching off the irradiation in the dark (dashed area "B"), is in the order of the number of chlorophyll molecules in the investigated suspensions. freely diffusing unstable photoproduct P. This implies that a short saturating flash induces the formation of a number of $\mathrm{P}$ equal to the number of chlorophyll molecules in the investigated sample. They have also assumed that the photoproduct $P$ is stabilized at the level of the Emerson and Arnold enzyme E, whose concentration is much lower than that of chlorophyll. Assuming that the turnover time of $\mathrm{E}$ is much longer than the lifetime of $\mathrm{P}$, the amount of oxygen evolved will be proportional to $0.25 \mathrm{E}$. This is the case with flash experiments - non-cooperative Kok mechanism. Under low irradiance conditions, all $\mathrm{P}$ could be stabilized and the production of $\mathrm{O}_{2}$ is with high quantum yield efficiency through the cooperative mechanism. Having in mind the above results, the functioning of the oxygen-evolving centres may be presented as follows: In the darkness, all oxygen-evolving centres (or all chlorophyll molecules!) accept $\mathrm{CO}_{2}$ molecules or $\mathrm{HCO}_{3}^{-}$anions. This statement is in agreement with the results of Emerson and Lewis (1941a, b), McAlister (1939), and with the data of Stemler (1977, 1980a, b). At low irradiance every chlorophyll molecule works as a part of the RC with low frequency depending on the frequency of the quanta absorbed. If the irradiance is sufficiently high, it leads to the oxidation (blocking) of a significant part of the oxygenevolving centres, a process connected with the $\mathrm{O}_{2}$ consumption which leads to $\mathrm{CO}_{2}$ evolution from the oxygen-evolving centres during the induction time of photosynthesis [the "first effect of Emerson" (Emerson and Lewis, 1941a, b)]. At saturating irradiance the number of the functionally effective (working or operative) oxygen-evolving centres can decrease to ca. 1:500, and thus the number of $\mathrm{O}_{2}$ molecules absorbed or $\mathrm{CO}_{2}$ molecules evolved during the induction time would be practically equal to the number of chlorophyll molecules in the investigated photosynthetic system ${ }^{1}$. This assertion explains the observed radia-

\footnotetext{
${ }^{1}$ Having in mind that the number of the oxygen molecules absorbed during the induction time of photosynthesis is equal to the number of the evolved $\mathrm{CO}_{2}$ molecules, we can reject Emerson's statement (Emerson and Lewis, $1941 \mathrm{a}, \mathrm{b}$ ) that the $\mathrm{CO}_{2}$ burst during the induction time is leading to a mistake in Warburg's experiments on the minimum quantum requirement of photosynthesis. It is evident that the evolved $\mathrm{CO}_{2}$ molecules simply replace the absorbed oxygen molecules! At the same time the process of blocking and unblocking of the oxygen-evolving centres during the
} 
tion dependence of the induction times. According to the interpretation presented above that the quanta arrive at the oxygen-evolving centres after prolonged intervals (longer than several seconds), the centres cannot reach the higher oxidized $\mathrm{S}_{3}$ or $\mathrm{S}_{4}$ states and oxygen can be evolved by the co-operation of oxygen precursors obtained in different centres, a mechanism considered previously (Zeinalov, 1982, 2005; Zeinalov and Maslenkova, 1996). In summary, the following reaction steps could be presumed:

$$
\begin{aligned}
& \mathrm{ChlZ}_{+} \mathrm{HCO}_{3}^{-} \rightarrow \mathrm{ChlZHCO}_{3}^{-}, \\
& \mathrm{ChlZHCO}_{3}^{-}+\mathrm{h} v \rightarrow \mathrm{Chl}^{*} \mathrm{ZHCO}_{3}^{-}, \\
& \mathrm{Chl}^{*} \mathrm{ZHCO}_{3}^{-} \rightarrow \mathrm{Chl}^{+} \mathrm{Z}^{-} \mathrm{HCO}_{3}^{-}, \\
& \mathrm{Chl}^{+} \mathrm{Z}^{-} \mathrm{HCO}_{3}^{-}+\mathrm{P} \rightarrow \mathrm{Chl}^{+} \mathrm{ZHCO}_{3}^{-}+\mathrm{P}^{-}, \\
& \mathrm{Chl}^{+} \mathrm{ZHCO}_{3}^{-} \rightarrow \mathrm{ChlZ}^{-} \mathrm{HCO}_{3}, \\
& 4 \mathrm{HCO}_{3} \rightarrow 2 \mathrm{H}_{2} \mathrm{O}+4 \mathrm{CO}_{2}+\mathrm{O}_{2}, \\
& \mathrm{H}_{2} \mathrm{O}+\mathrm{CO}_{2}+\mathrm{CA} \rightarrow \mathrm{H}_{2} \mathrm{CO}_{3} \rightarrow \\
& \mathrm{H}^{+}+\mathrm{HCO}_{3}^{-}, \\
& \mathrm{ChlZHCO}_{3}^{-}+\mathrm{O}_{2}+\mathrm{P}_{1}+\mathrm{h} v \rightarrow \\
& \mathrm{ChlZO}_{2}+\mathrm{HCO}_{3}+\mathrm{P}_{1}^{-}, \\
& 4 \mathrm{HCO}_{3} \rightarrow 2 \mathrm{H}_{2} \mathrm{O}+4 \mathrm{CO}_{2}+\mathrm{O}_{2}, \\
& \mathrm{ChlZO}_{2}+\mathrm{HCO}_{3}^{-} \rightarrow \mathrm{ChlZHCO}_{3}^{-}+\mathrm{O}_{2} .
\end{aligned}
$$

During the first reaction (8) the oxygen-evolving centres (i.e. all chlorophyll molecules!) capture a bicarbonate ion in the darkness. The second reaction (9) reflects the light quanta absorption by the chlorophyll molecule, which is in complex with the primary electron acceptor $(Z)$. During (10) the charges separation is accomplished and one electron is transferred from the excited chlorophyll molecule to $Z$. The next reaction (11) is the electron transfer to a component $\mathrm{P}$ on the electron transport chain. The electron of the bicarbonate ion fills the missing electron in the chlorophyll molecule and the bicarbonate ion is separated as a bicarbonate radical [reaction (12)]. The recombination of four bicarbonate radicals [reaction (13)], accumulated at a given reaction centre (in flash experiments or under high irradiance conditions), leads to the evolution of one oxygen molecule, two molecules of water and four $\mathrm{CO}_{2}$ molecules - the so-called non-cooperative or

induction time could be realized as "one quantum process", and could be used for interpretation of "the one quantum" requirement reaction by Warburg, as it was mentioned by Vennesland (1965). Obviously, the $\mathrm{CO}_{2}$ and $\mathrm{O}_{2}$ exchange processes during the induction time of photosynthesis need further profound investigations using modern inertialess experimental techniques.
Kok's oxygen-evolving mechanism. Under low irradiances or after switching off the irradiance, the cooperation of the four bicarbonate radicals, produced in different reaction centres leads again to the same reaction - cooperative mechanism. The restored complex of the chlorophyll molecule, the primary acceptor in reaction (12) and the restored $\mathrm{CO}_{2}$ molecules [reaction (13)], after hydration with the participation of carboanhydrase CA [reaction (14)], are involved in (8) and the cycle could start again.

Reaction (15) takes place under irradiation and increased oxygen concentration during the induction time of photosynthesis and is connected with the inactivation (blocking) of the oxygen-evolving centres. The process is connected with the liberation of bicarbonate radicals, after the recombination of which [reaction (16)] the process of the $\mathrm{CO}_{2}$ burst (Emerson and Lewis, 1941a, b) is accomplished. In summary, these two reactions lead to oxygen absorption and $\mathrm{CO}_{2}$ liberation ${ }^{2}$. Finally during (17) the oxygen liberation and $\mathrm{HCO}_{3}^{-}$ catching from the blocked oxygen-evolving centres after switching the light off occurs and the system returns to the initial state (8). Most likely, the possible path of cooperation of the four bicarbonate radicals in (13) and (16) is accomplished, according to the hypothesis of Metzner (1978), through dimerization of two bicarbonate radicals

\footnotetext{
${ }^{2}$ Here it is necessary to point out that the chlorophyll in the blocked $\mathrm{ChlZO}_{2}$ state in reaction (15) is most probably in a high fluorescent state, and the generally accepted interpretation of the fluorescent induction phenomena using modulated PAM techniques as "photochemical", "non-photochemical" quenching should be reconsidered and revised. The low fluorescent $\mathrm{F}_{0}$ state could be logically connected with the initial oxygen burst observed during the induction time of photosynthesis when all oxygen-evolving centres (i.e. all chlorophyll molecules which are in the $\mathrm{ChlZ}_{\mathrm{HCO}}{ }_{3}^{-}$state) are in the open state. The following increase of the fluorescence, and reaching of the $\mathrm{F}_{m}$ level, could be connected with the accumulation of the blocked $\mathrm{ChlZO}_{2}$ state. And finally the subsequent decrease and reaching of the steady state most likely is connected with the mechanical movement (phototaxis) of the cells, chloroplasts and thylakoids, leading to the more effective capturing of the light quanta. Thus the blocking process of the oxygen-evolving centres (15) decreases the number of effectively working reaction centres, i.e. the level of oxygen concentration in the cell and chloroplast volumes, while simultaneously converting the working reaction centres in an effectively fluorescent state $\left(\mathrm{ChlZO}_{2}\right)$, protecting in this way the photosynthetic systems from photodamage.
} 
to peroxidicarbonic acid. In fact (15) regulates the rate of photosynthesis (oxygen evolution) by the so-called "negative feedback" regulation and probably gives an idea for interpretation of the complexity of the registered oxygen induction phenomena, a problem with a long history (see Rabinowitch, 1951, Chapter XXXIII) and without an answer yet. So, instead of the enzyme regulation, accepted by Franck and Herzfeld (1941), our results reflecting the variation of the oxygenevolving centres suggest negative feedback regulation of the process of photosynthesis or oxygen evolution. In the same time the reaction (15) gives a logical interpretation of the "first effect of Warburg", i.e. the inhibitory effect of oxygen on photosynthesis. The presented results show that the initial oxygen burst is a result of two opposite processes - oxygen production from the enormous (equal or close to the number of chlorophyll molecules in the investigated algae suspension) number of oxygen-evolving centres (all oxygen-evolving centres are in an open, reactivated in the preceding dark incubation time state) and the process of oxygen consumption, connected with the process of blocking the essential part of the reaction centres. If the process of blocking does not exist, the stationary oxygen evolution rate could reach extremely high levels (a value at least one hundred times bigger) and the photosynthesizing system could be submitted to self-destruction by increased oxygen concentration. This is the reason for the extremely fast blocking process (see Fig. 7). Apparently, if the reactions presented above reflect the molecular events in the oxygen-evolving centres, the isotopic experiments with labeled oxygen will reveal the water as a source of photosynthetic oxygen. The water is included as the ultimate source of electrons in reaction (14) during hydration of $\mathrm{CO}_{2}$. It is also clear that $\mathrm{CO}_{2}$ is included in the reaction sequence only as a catalyst. A more detailed consideration of the presented reaction scheme could show an analogy between the complex ChlZH$\mathrm{CO}_{3}{ }^{-}$and the so-called "photolyte" component of the celebrated Otto Warburg (Warburg 1920; see also Höxtermann, 2007; Nickelsen, 2007). According to Warburg et al. (1969) the binding of carbonic acid occurs in two consecutive reactions, both of which require the energy provided by oxygen respiration. The first reaction leads to aerobically bound carbonic acid; the second reaction, which is catalyzed by manganese (Warburg et al., 1968), leads to the photolyte (Warburg and Krippahl, 1967) in which one molecule of carbonic acid is combined with one molecule of chlorophyll. In reality this component formed in the dark [reaction (8) during the reaction sequence (8)-(13) leads to the evolution of one oxygen molecule through the electrolysis of $\mathrm{H}_{2} \mathrm{CO}_{3}$ ]. Since this electrolysis runs to account for the absorbed quanta of energy, it could be named photo-electrolysis.

In summary, the general equation of photosynthesis should be presented as

$$
\begin{aligned}
& \mathrm{CO}_{2}+\mathrm{H}_{2} \mathrm{O}+\mathrm{CA} \rightarrow \mathrm{H}_{2} \mathrm{CO}_{3} \rightarrow \\
& \mathrm{H}^{+}+\mathrm{HCO}_{3}^{-}+\mathrm{h} v \rightarrow \mathrm{CH}_{2} \mathrm{O}+\mathrm{O}_{2} .
\end{aligned}
$$

In this scheme, the first two steps are dark reactions without using the energy of light quanta. These two steps accumulate from the surrounding media 3.9 and $8.6 \mathrm{kcal} / \mathrm{mol}$ energy, respectively (Dismukes et al., 2001). While the entire reaction needs $45.9 \mathrm{kcal} / \mathrm{mol}$, the use of the bicarbonate ions decreases the light reaction energy to $33.4 \mathrm{kcal} / \mathrm{mol}$ (approx. 1.37 times).

The above interpretation explains the results presented in Fig. 2. The induction curves show that the duration of the induction period decreases simultaneously with decreasing irradiance, and under low intensity $\left(0.056 I_{0}\right)$ the rate of oxygen evolution reaches its steady state immediately after switching on the light - reactions (15) and (16) cannot be accomplished as the concentration of oxygen in the suspension or chloroplast volumes is low (low irradiation!).

The concept of the "photosynthetic unit" is now more than 75 years old. During this period, our ideas about the size and the arrangement of these structures have often been changed. The most difficult question, however, still remains: "Are there other possibilities for the explanation of the existing observations?" I suppose that if Emerson and Arnold (1932a, b) and Gaffron and Wohl (1936) had had in their possession the results presented in Figs. 3-8 which show dramatic changes in the number of operative oxygen-evolving centres during the induction time, it could hardly be assumed that they would have postulated their hypothesis about the PSU. Unfortunately, all their experiments were performed with Warburg's manometric apparatus. Using this equipment, they could not observe the oxygen yields by separate flashes, i.e. they could not observe any changes in the number of operative oxygen-evolving centres. Emerson and 
Arnold (1932a, b) measured only the oxygen yield from a great number of flashes and divided it by the number of generated flashes to calculate the yields by separated flashes. Without having any indications about the extremely fast (taking only tenths of a second) process of blocking of the oxygen-evolving centres, they simply have supposed that the number of operating reaction centres remains constant during the induction time of photosynthesis. The results presented above show the complexity and flexibility of the oxygen-evolving system of photosynthesis. They demonstrate that many of the experimental data obtained cannot be understood in the frame of the postulated concept of the PSU. Furthermore, there are many observations, the explanation of which leads to serious contradictions; meanwhile, they are the reason for the proposal of various models. Regarding the basic arguments for the postulation of a PSU we have to admit that the strongest point is the absence of oxygen after the first saturating flash. However, this demands a very careful reconsideration: after prolonged darkness the first flash hits the cells or the chloroplasts in an anaerobic state; the rate constants of the reactions leading to $\mathrm{O}_{2}$ evolution through the cooperative mechanism are significantly lower than those connected with a non-cooperative mechanism, since the functioning of a cooperative mechanism requires the diffusion of $\mathrm{O}_{2}$ precursors between different RCs. Photosynthetic systems are self-controlled and may attain a modified state after a short saturating flash. This may be connected with $\mathrm{O}_{2}$ consuming processes during the induction period [reactions (15) and (16)] and furthermore connected with self-regulating processes, which protect the living structure from oxidative damage. This statement is supported by the data of Boitchenko and Efimtcev (1979) showing that at increased oxygen concentrations a significant part of the oxygen-evolving (PSII) centres are inactivated (blocked).

Therefore, all three basic arguments about the PSU concept could be explained by the existence of two different ways of oxygen evolution in photosynthesis and by the different degree of inactivation (blocking!) of the oxygen-evolving centres. In this respect, the concept of the PSU should rather be accepted as a dynamic than as a structural or statistical system ${ }^{3}$.

The acceptance of a dynamic system with respect to the functional properties and potential ability of every chlorophyll molecule to perform the initial photochemical charge separation reaction eliminates all artificially created problems and preserves all remaining characteristics of the structural or statistical ideas about the PSU. This idea is in agreement with the statements of Vennesland (1965) according to which "the 1:1:1 molar equivalent relation of the aerobically bound $\mathrm{CO}_{2}$ :glutamate:chlorophyll shows that all of these components contribute to the activated complex which functions as an $\mathrm{O}_{2}$ precursor when it absorbs light." And "This is the photosynthetic unit of Warburg. It contains one molecule of chlorophyll-not one hundred."

The most serious and difficult problem, arising from the original concept of the PSU, is connected with the mechanisms of the directed energy transfer from the antenna chlorophyll molecules to the respective reaction centres. It is evident that the only possible mechanism for such kind of energy transfer from 200-300 chlorophyll molecules, from the light-harvesting complexes to the specialized reaction centres is the existence of a demon like Maxwell's demon in thermodynamics. On the contrary, the concept of the dynamic system suggests that every chlorophyll molecule is in a state to perform the charge separation reaction and the problem of energy migration between hundreds of molecules does not exist. Under low irradiance conditions, the separated chlorophyll molecules absorb light quanta with low frequency (after long dark intervals) and are not submitted to inactivation or blocking processes. Under such conditions, every chlorophyll molecule functions as a reaction centre and the process of oxygen production is realized through the cooperative oxygen-evolving mechanism. On the other hand,

\footnotetext{
${ }^{3}$ The crude calculations show that the kinetics of the restoration of the blocked reaction centres $\left(\mathrm{ChlZO}_{2}\right)$ according to equation (17), presented in Figs. 5-8, leads to the activation of only one centre in $600 \mathrm{chlo-}$ rophyll molecules for 10-20 ms. Thus we could admit that during the steady-state photosynthesis there exists a dynamic equilibrium between open (functionally active) and blocked reaction centres. Obviously, further investigations are needed for the understanding of the real events of the photosynthetic oxygen production.
} 
under high irradiance conditions, when the essential part of the chlorophyll molecules is in a blocked state and only a small part is in a functionally active state as a consequence of the high concentration of the incident quanta, every chlorophyll molecule $\left(\mathrm{ChlZHCO}_{3}^{-}\right.$or better ChlGl$\left.\mathrm{HCO}_{3}^{-}\right)$in an active state could absorb light quanta with sufficiently high frequency. This does not mean that energy migration is forbidden or not existing. According to the known physical mechanisms the energy transfer is realized, however, its role and significance for the primary photophysical and photochemical processes could be ignored in comparison with the direct absorption of light quanta from the functionally active reaction centres. In other words, the energy transfer is accomplished as in solid or liquid media, without any "directed transfer" towards the "highly specialized" and strictly determined "reaction centre" molecules.

The essential conclusion, based on the presented results and their analysis, is that the number of oxygen-evolving centres under low irradiance conditions tends to the number of chlorophyll molecules in the photosynthesizing systems. Under such conditions, oxygen evolution is mainly a result of the so-called cooperative oxygen-evolving mechanisms, accomplished by PSII $\beta$ centres (situated in the stroma thylakoid regions). The second component of oxygen evolution is connected with the deactivation of the higher oxidized $\left(\mathrm{S}_{2}\right.$ or $\left.\mathrm{S}_{3}\right)$ states from grana domains (PSII $\alpha$ centres). With increasing light intensity, the process of blocking of the reaction centres starts, according to (15), a process connected with the oxygen consumption and $\mathrm{CO}_{2}$ evolution [cf. (16)] which explains Emerson's first effect or the $\mathrm{CO}_{2}$ burst during the induction time of photosynthesis. Under saturated irradiance conditions, the number of the effectively working centres decreases to the number estimated by Emerson and Arnold [1RC/2500 chlorophyll molecules (big PSUs) or 1RC/600 chlorophyll molecules].
The ideas and the results presented in the present work explain several experimental observations (some of them considered as "effects") unexplained up to now: a) the linearity of the photosynthetic light curves (without the effect of respiration!) and the absence of the prolonged induction time under low irradiance conditions with the participation of the cooperative oxygenevolving mechanism; b) the oscillations of the oxygen flash yield amplitudes with the participation of the non-cooperative (Kok's) mechanisms under flash (saturating!) irradiation; c) the oxygen consumption and $\mathrm{CO}_{2}$ burst during the induction time of photosynthesis with the blocking and inhibition of the oxygen-evolving centres of photosynthesis ["oxygen effect" of Warburg (1920) and "first effect of Emerson" (Emerson and Lewis, 1941a, b)].

At the same time, the problems of energy migration from antenna structures to the "specialized reaction centres" will significantly diminish. Under low irradiance conditions, as it was pointed out, every chlorophyll molecule, after absorption of light quanta, will act as a reaction centre. On the other hand, under higher irradiances (days of sunlight $-1500-1800 \mu \mathrm{mol} \mathrm{h} v \mathrm{~m}^{-2} \mathrm{~s}^{-1}$ ) the chlorophyll molecules remaining in an active state (unblocked) with an $0.2 \cdot 10^{-16} \mathrm{~cm}^{2}$ average effective cross section for the light quanta absorption (in solutions!) will catch one light quantum within an interval below $500 \mathrm{~ms}$. Having in mind that in biological systems (leaves, unicellular algae etc.) the effective cross section for quanta absorption of the individual chlorophyll molecules can increase significantly as a consequence of light scattering, the intervals of the quanta absorption could access to the turnover time [closed time of reaction centres (Emerson and Arnold period)] of the oxygen-evolving centres. Consequently, under such conditions, energy migration will not be needed from the so-called antenna chlorophyll molecules or from another kind of "light-harvesting complexes" to the specialized, so-called "reaction centres" molecules. 
Alen F. L. and Franck J. (1955), Photosynthetic evolution of oxygen by flashes of light. Arch. Biochem. Biophys. 58, 124-143.

Boitchenko V. A. and Efimtcev E. I. (1979), Ingibirovanie aktivnosti fotosystemi II u Chlorelli pri visokih koncentracii kisloroda (Inhibition of the PSII activity in Chlorella under high $\mathrm{O}_{2}$ concentrations). Fiziol. Rastenii 26, 815-823 (in Russian).

Delrieu M.-J. (1981), 3-(3,4-Dichlorphenyl)-1,1-dimethylurea effects on the oxidizing side of photosystem II. Photobiochem. Photobiophys. 3, 137-144.

Dismukes G. C., Klimov V. V., Baranov S. V., Kozlov Y. N., Das Gupta J., and Tyryshkin A. (2001), The origin of atmospheric oxygen on earth: The innovation of oxygenic photosynthesis. PNAS 98, 2170-2175.

Emerson R. and Arnold W. (1932a), A separation of the reactions in photosynthesis by means of intermittent light. J. Gen. Physiol. 15, 391-420.

Emerson R. and Arnold W. (1932b), The photochemical reaction in photosynthesis. J. Gen. Physiol. 16, 191-205.

Emerson R. and Lewis C. M. (1941a), Carbon dioxide exchange and the measurement of the quantum yield of photosynthesis. Am. J. Bot. 28, 789-804.

Emerson R. and Lewis C. M. (1941b), The quantum efficiency of photosynthesis. Carnegie Inst. Yearbook 40, 157-160.

Franck J. and Herzfeld K. F. (1941), Contribution to a theory of photosynthesis. J. Phys. Chem. 45, $978-1025$.

Gaffron H. and Wohl K. (1936), Zur Theorie der Assimilation. Naturwissenschaften 24, 81-103.

Höxtermann E. (2007), A comment on Warburg's early understanding of biocatalysis. Photosynth. Res. 92, $121-127$.

Joliot P. (1961), Cinetique d'induction de la photosynthese chez Chlorella pyrenoidosa. II. Cinetique d'emission d'oxygene et fluorescence pendant la phase d'illumination. J. Chim. Phys. 58, 584-595.

Joliot P., Barbieri G., and Chabaud R. (1969), Un nouveau modele des centre photochimique du systeme II. Photochem. Photobiol. 10, 309-329.

Kok B., Forbush B., and McGloin M. (1970), Co-operation of charges in photosynthetic $\mathrm{O}_{2}$ evolution. I. A linear four step mechanism. Photochem. Photobiol. 11, 457-475.

Lavorel J. (1976), Matrix analysis of the oxygen evolving system of photosynthesis. J. Theor. Biol. 57, $171-185$

Lavorel J. (1980), On the origin of damping of the oxygen yield in sequences of flashes. In: Photosynthetic Oxygen Evolution (Metzner H., ed.). Academic Press, New York, pp. 249-268.

Lavorel J. and Joliot P. (1972), A connected model of the photosynthetic unit. Biophys. J. 12, 815-831.

Lavorel J. and Lemasson C. (1976), Anomalies in the kinetics of photosynthetic oxygen emission in sequences of flashes revealed by matrix analysis. Effect of carbonyl cyanide $m$-chlorophenylhydrazone and variation in time parameters. Biochim. Biophys. Acta 430, 501-516.

Lavorel J. and Maison-Peteri B. (1983), Studies of deactivation of the oxygen-evolving system in higher plant photosynthesis. Physiol. Veg. 21, 509-517.
Lehoczki E. and Zeinalov Y. (1984), Unusual photosynthetic oxygen evolution. I. Cerulenin-induced 3-(3,4-dichlorophenyl)-1,1-dimethylurea insensitive oxygen evolution in Chlorella pyrenoidosa. Photobiochem. Photobiophys. 7, 135-142.

Maslenkova L. T., Zanev (Zeinalov) Y., and Popova L. P. (1989), Effect of abscisic acid on the photosynthetic oxygen evolution in barley chloroplasts. Photosynth. Res. 21, 45-50.

Mauzerall D. and Greenbaum N. L. (1989), The absolute size of a photosynthetic unit. Biochim. Biophys. Acta 974, 119-140.

McAlister E. D. (1939), The chlorophyll-carbon dioxide during photosynthesis. J. Gen. Physiol. 22, 613-636.

Metzner H. (1978), Oxygen evolution as energetic problem. In: Photosynthetic Oxygen Evolution (Metzner H., ed.). Academic Press, New York, pp. 59-76.

Nickelsen K. (2007), Otto Warburg's first approach to photosynthesis. Photosynth. Res. 92, 109-120.

Rabinowitch E. (1951), Photosynthesis and Related Processes, Vol 2. Interscience Publishers Inc., New York.

Schmidt G. H. and Gaffron H. (1971), Fluctuating photosynthetic units in higher plants and fairly constant units in algae. Photochem. Photobiol. 14, 451-464.

Scilard A., Sass L., and Vass I. (2002), Photoinactivation of photosystem II at low light intensity. Mathematical models. Acta Biologica Szegediensis 46, 167-169.

Shinkarev V. P. (2005), Flash-induced oxygen evolution in photosynthesis: Simple solution for the extended S-state model that includes misses, double hits, inactivation, and backward-transitions. Biophys. J. 88, $412-421$.

Stemler A. (1977), The binding of bicarbonate ions to washed chloroplast grana. Biochim. Biophys. Acta 560, 511-522.

Stemler A. (1980a), Inhibition of photosystem II by formate. Possible evidence for a direct role of bicarbonate in photosynthetic oxygen evolution. Biochim. Biophys. Acta 593, 103-112.

Stemler A. (1980b), Forms of dissolved carbon dioxide required for photosystem II activity in chloroplast membranes. Plant Physiol. 65, 1160-1165.

Tumerman L. A. and Sorokin E. M. (1967), Fotosyntheticheskaya edinitsa: "Fizicheskaya" ili "Statisticheskaya" model? (Photosynthetic unit: "Physical" or "statistical" model?). Mol. Biol. 1, 628-638 (in Russian).

Vennesland B. (1965), The energy conversion reactions of photosynthesis. In: Biochemical Dimensions of Photosynthesis (Krogmann D.W. and Powers W.H., ed.). Wayne State University Publishers, Detroit, pp. 48-61.

Warburg O. (1920), Über die Geschwindigkeit der photochemischen Kohlensäurezersetzung in lebenden Zellen. Biochem. Z. 103, 188-217.

Warburg O. and Krippahl G. (1967), Photolyt und Chlorophyll in Chlorella. Biochem. Z. 346, 418-428.

Warburg O., Krippahl G., and Lehman A. (1968), Dialysierte Chlorella, ein neues Versuchsmaterial zur Untersuchung der Photosynthese. Z. Naturforsch. 23b, 1076-1079.

Warburg O., Krippahl G., and Lehman A. (1969), Chlorophyll catalysis and Einstein's law of photochemi- 
cal equivalence in photosynthesis. Am. J. Bot. 56, 961-971.

Whittingham C. P. and Brown A. H. (1958), Oxygen evolution from algae illuminated by short and long flashes of light. J. Exp. Bot. 9, 311-319.

Zeinalov Y. (1977), Non-additiveness in the action of light at the photosynthesis of green plants. Compt. Rend. Acad. Bulg. Sci. 30, 1479-1482.

Zeinalov Y. (1979), On the amount of oxygen taken up during the induction period of photosynthesis in green algae. Compt. Rend. Acad. Bulg. Sci. 32, $679-682$.

Zeinalov Y. (1982), Existence of two different ways for oxygen evolution in photosynthesis and photosynthetic unit concept. Photosynthetica 16, 27-35.

Zeinalov Y. (1986), What does "photosynthetic unit" mean? Photobiochem. Photobiophys. 11, 151-157.
Zeinalov Y. (2002), An equipment for investigations of photosynthetic oxygen production reactions. Bulg. J. Plant Physiol. 28, 57-67.

Zeinalov Y. (2005), Mechanisms of photosynthetic oxygen evolution and fundamental hypotheses of photosynthesis. In: Handbook of Photosynthesis, 2nd ed. (Pessarakli M., ed.). CRC Press, Taylor \& Francis Group, Boca Raton, FL, pp. 3-19.

Zeinalov Y. and Litvin F. F. (1979), Oxygen evolution after switching off the light and Si-state deactivation in photosynthesizing systems. Photosynthetica 13, $119-123$

Zeinalov Y. and Maslenkova L. (1996), Mechanisms of photosynthetic oxygen evolution. In: Handbook of Photosynthesis (Pessarakli M., ed.). Marcel Dekker, New York, pp. 129-150. 